\title{
Edaravone inhibits pressure overload-induced cardiac fibrosis and dysfunction by reducing expression of angiotensin II ATI receptor
}

This article was published in the following Dove Press journal:

Drug Design, Development and Therapy

16 October 2017

Number of times this article has been viewed

\author{
Wei-Wei Zhang ${ }^{1,2}$ \\ Feng Bai' \\ Jin Wang' \\ Rong-Hua Zheng' \\ Li-Wang Yang' \\ Erskine A James ${ }^{3}$ \\ Zhi-Qing Zhao ${ }^{1,4}$
}

'Department of Physiology, Shanxi Medical University, ${ }^{2}$ Department of Anesthesiology, Shanxi Provincial People's Hospital, Taiyuan, Shanxi, China; ${ }^{3}$ Department of Internal Medicine, Navicent Health, Macon, ${ }^{4}$ Department of Basic Biomedical Sciences, Mercer University School of Medicine, Savannah, GA, USA
Correspondence: Zhi-Qing Zhao Department of Basic Biomedical Sciences, Mercer University School of MedicineSavannah Campus, 1250 East 66th Street, Savannah, GA 3I404, USA

$\mathrm{Tel}+|9| 272 \mid 8208$

Fax + I 91272I 8268

Email zhao_z@mercer.edu
Abstract: Angiotensin II (Ang II) is known to be involved in the progression of ventricular dysfunction and heart failure by eliciting cardiac fibrosis. The purpose of this study was to demonstrate whether treatment with an antioxidant compound, edaravone, reduces cardiac fibrosis and improves ventricular function by inhibiting Ang II AT1 receptor. The study was conducted in a rat model of transverse aortic constriction (TAC). In control, rats were subjected to 8 weeks of TAC. In treated rats, edaravone $(10 \mathrm{mg} / \mathrm{kg} /$ day $)$ or Ang II AT1 receptor blocker, telmisartan $(10 \mathrm{mg} / \mathrm{kg} /$ day $)$ was administered by intraperitoneal injection or gastric gavage, respectively, during TAC. Relative to the animals with TAC, edaravone reduced myocardial malonaldehyde level and increased superoxide dismutase activity. Protein level of the AT1 receptor was reduced and the AT2 receptor was upregulated, as evidenced by the reduced ratio of AT1 over AT2 receptor $(0.57 \pm 0.2$ vs $3.16 \pm 0.39, p<0.05)$ and less locally expressed AT1 receptor in the myocardium. Furthermore, the protein level of angiotensin converting enzyme 2 was upregulated. In coincidence with these changes, edaravone significantly decreased the populations of macrophages and myofibroblasts in the myocardium, which were accompanied by reduced levels of transforming growth factor beta 1 and Smad2/3. Collagen I synthesis was inhibited and collagen-rich fibrosis was attenuated. Relative to the TAC group, cardiac systolic function was preserved, as shown by increased left ventricular systolic pressure (204 \pm 51 vs $110 \pm 19 \mathrm{mmHg}, p<0.05)$ and ejection fraction $(82 \% \pm 3 \%$ vs $60 \% \pm 5 \%, p<0.05)$. Treatment with telmisartan provided a comparable level of protection as compared with edaravone in all the parameters measured. Taken together, edaravone treatment ameliorates cardiac fibrosis and improves left ventricular function in the pressure overload rat model, potentially via suppressing the AT1 receptor-mediated signaling pathways. These data indicate that edaravone might be selected in combination with other existing drugs in preventing progression of cardiac dysfunction in heart failure.

Keywords: angiotensin II receptor, cardiac fibrosis, cardiac function, edaravone, heart failure

\section{Introduction}

Cardiac fibrosis develops in response to various cardiovascular diseases including persisting hypertension, hypertropic cardiomyopathy, ischemic insults, and congenital heart defects. ${ }^{1,2}$ Pathogenesis of cardiac fibrosis involves cellular and molecular alterations in the myocardium, characterized by macrophage accumulation, fibroblast differentiation, and excessive diffuse collagen deposition in the interstitial and perivascular regions, and is associated with the development of arrhythmias, myocardial dysfunction, and heart failure. ${ }^{3,4}$ The approaches that reduce cardiac fibrosis have been shown to prevent progression of heart failure and many other cardiovascular diseases. ${ }^{5,6}$ 
It is well known that angiotensin II (Ang II), converted from angiotensin I by angiotensin converting enzyme (ACE), has profound effects on cardiac fibrosis via modulating the expression of the Ang II receptors. By stimulating the AT1 receptor, Ang II causes vascular constriction, systemic inflammatory response, interstitial collagen deposition, and tissue fibrosis, while the stimulation of the AT2 receptor counteracts the deleterious effects of the AT1 receptor. ${ }^{7-9}$ On the other hand, angiotensin converting enzyme 2 (ACE2), a newly found homolog of ACE, converts Ang II to Ang (1-7) to offset the function of Ang II. ${ }^{10,11}$ Our laboratory has had a longstanding interest in the areas of Ang II-induced fibrosis and cardiac dysfunction. We have previously reported that pharmacological inhibition of the AT1 receptor significantly reduces cardiac fibrosis and improves cardiac function. ${ }^{4,6}$ Although ACE inhibitors (ACEi) or AT1 receptor blockers (ARBs) have been well documented as treatment options for patients with hypertension and cardiovascular diseases, limitations associated with the use of these drugs have also been identified (eg, dry cough, angioedema, and hypotensive symptoms). In addition to their potential side effects, the responses to ACEi treatment are influenced by gender and ethnic diversity, and lesser efficacy of ARBs is reported in the treatment of end-organ damage. ${ }^{12,13}$

Edaravone (brand names, Radicava or Radicut; Mitsubishi Pharma, Japan), a free radical scavenger, was initially approved to help with recovery following an acute ischemic stroke in Japan in 2001. ${ }^{14}$ It has also been approved by the Food and Drug Administration in the United States to treat patients with amyotrophic lateral sclerosis in $2017 .{ }^{46}$ Edaravone acts as a potent antioxidant to scavenge free radicals, inhibit oxidative stress, and protect the brain against neuronal apoptosis. Furthermore, it aids neurological recovery following acute brain ischemia and subsequent cerebral infarction. ${ }^{15}$ Many clinical and experimental studies have demonstrated that edaravone exerts beneficial effects on the heart against ischemia/reperfusion injury, ${ }^{16}$ cardiomyocyte hypertrophy, ${ }^{17}$ and tissue fibrosis in dilated cardiomyopathy, ${ }^{18}$ primarily through trapping and quenching reactive oxygen species (ROS). Although many studies have identified edaravone as a potent antioxidant compound in cardioprotection, it is unknown whether edaravone has a direct effect on the pathogenesis of Ang II-initiated cardiac injury. Therefore, in the present study, we tested the hypothesis that edaravone reduces cardiac fibrosis and improves cardiac function via inhibiting Ang II AT1 receptor-activated signaling. We selected a rat model of transverse aortic constriction (TAC) to investigate the therapeutic effects of edaravone on expression of the AT1/AT2 receptors and ACE2, activation of oxidative stress, accumulation of macrophages, proliferation of myofibroblasts, and deposition of collagen. To further demonstrate whether the effect of edaravone on expression of the AT1 receptor is responsible for protection, the AT1 receptor antagonist, telmisartan, was selected for comparison. In coincidence with these measurements, cardiac fibrosis was measured morphologically and cardiac function was detected by echocardiography.

\section{Materials and methods Animals and TAC model}

Sprague Dawley rats at 6-8 weeks of age (Animal Laboratory Center of Shanxi Medical University, Taiyuan, China) were used in the present study. The animal experimental procedures were approved by the Institutional Animal Care and Use Committee, Shanxi Medical University, in compliance with The Guide for the Care and Use of Laboratory Animals published by the National Institute of Health USA. ${ }^{47}$ Rats were housed under standard conditions (12-hour light/dark cycle, temperature at $20^{\circ} \mathrm{C}-25^{\circ} \mathrm{C}$, and relative humidity at $50 \%-60 \%$ ). No significant differences in age and body weight were found among all the rats before surgery. TAC was produced according to methods described previously with minor modifications. ${ }^{19}$ In brief, rats were anesthetized with an intraperitoneal injection of a mixture of ketamine (90 mg/kg) and zylaxine (10 mg/kg) (Henry Schein, Inc., Indianapolis, IN, USA) and placed on a temperature controlled surgical table. Under sterile conditions, the chest was opened and the aorta was carefully dissected. A bended and blunt stylet from a $18 \mathrm{G}$ intravenous catheter was tied tightly to the aorta between the brachiocephalic trunk and the left common carotid artery using a 4-0 silk suture, and then pulled out to create partial aortic constriction. Procedures on the first surgical day were performed under sterile condition. After surgery, rats were kept in a preheated chamber until they fully recovered from anesthesia, and then moved to individual cages, where they were kept for an 8-week experimental period.

\section{Experimental protocol and group}

The rats were randomly divided into four groups ( $\mathrm{n}=6$ in each group) after surgery: 1) sham operation (Sham) - rats underwent the same surgical procedure without banding the aorta; 2) TAC - rats were subjected to TAC for 8 weeks; 3) TAC plus edaravone (Edara; Boda Pharmaceutical Co, Ltd, Jilin, China) - rats received an intraperitoneal injection 
of edaravone at a dose of $10 \mathrm{mg} / \mathrm{kg} /$ day after TAC; 4) TAC plus telmisartan (Telmi) - rats were administered telmisartan (Boehringer Ingelheim Pharmaceuticals, Inc, Ridgefield, CT, USA) via gastric gavage at a dose of $10 \mathrm{mg} / \mathrm{kg} /$ day after TAC. The doses selected for edaravone and telmisartan were based on previous experiments, which have shown a significant reduction in oxygen radical and decrease in blood pressure. ${ }^{4,17,18}$

\section{Measurement of the lipid peroxidation level and antioxidant enzyme activity}

The hearts were homogenized in $20 \mathrm{mmol} / \mathrm{L}$ phosphate buffer as previously described. ${ }^{17}$ The level of malonaldehyde (MDA) in the left ventricle was measured as the index of lipid peroxidation with an MDA detection kit (Jiancheng Bioengineering Institute, Nanjing, China) and expressed as nmol/mg tissue according to the manufacturer's instructions. Superoxide dismutase (SOD) activity to represent the ability of trapping oxygen radicals was determined with a SOD detection kit (Jiancheng Bioengineering Institute) and measured as $\mathrm{U} / \mathrm{mg}$ tissue.

\section{Determination of heart/body weight ratio and myocyte sectional area (MSA)}

At the end of the experiment, the chest was opened and the heart was rapidly removed. The heart-body weight index (HW/BW) was calculated as heart weight divided by body weight ( $\mathrm{mg} / \mathrm{g}$ tissue). MSA was quantitatively analyzed after $6 \mu \mathrm{m}$ of tissue slides were stained with hematoxylin and eosin. Fifty myocytes were randomly selected and captured with a digital camera through $40 \mathrm{X}$ objective lens under light microscopy. Cardiomyocytes were defined as having a visible nucleus and intact cellular membrane. Eight randomized high-powered fields per tissue section were selected to measure MSA in series using an image software (ImageJ; $\mathrm{NIH}$, Rockville, MD, USA) and expressed as $\mathrm{mm}^{2} \times 10^{-4}$ of the mean.

\section{Protein levels of ATI/AT2 receptors, transforming growth factor beta I (TGF $\beta$ I), Smads, and collagen III by Western blotting}

Freshly frozen transmural tissue samples obtained from the different groups were homogenized in ice-cold lysis buffer. Protein concentration was measured by the DC protein assay method as previously reported. ${ }^{4}$ In brief, proteins $(60 \mu \mathrm{g})$ were separated by gradient sodium dodecyl sulfate-polyacrylamide gel electrophoresis and identified with the following antibodies to quantify their protein levels: rabbit anti-AT1 and AT2 receptor polyclonal antibodies (Santa Cruz Biotechnology Inc, Dallas, TX, USA), a mouse anti-TGF $\beta 1$ monoclonal antibody (Abcam, Cambridge, UK), rabbit anti-Smad2, 3, 4, and 7 monoclonal antibodies (Cell Signaling Technology, Danvers, MA, USA), a mouse anticollagen type III monoclonal antibody (Sigma-Aldrich Co, St Louis, MO, USA), and a mouse anti-beta actin monoclonal antibody (Sigma-Aldrich Co), respectively. After incubation with the primary antibody, the bound antibody was visualized with respective horseradish peroxidase-coupled secondary antibody. The membrane was then incubated with chemiluminescence developing agents and exposed to an $\mathrm{X}$-ray film. The actin level was determined in every sample. Films were scanned and band densities were quantified with densitometric analysis using the ImageJ system (NIH). The final band density was normalized with actin.

\section{Immunohistochemical staining for collagen I, ACE2, macrophages, and myofibroblasts}

The hearts were immersion-fixed in $10 \%$ buffered formalin and tissue blocks were embedded into paraffin. Samples were sectioned to $6 \mu \mathrm{m}$ thickness using a microtome (Leica RM2135; Meyer Instruments, Houston, TX, USA). After deparaffinization and hydration as previously reported, ${ }^{4}$ the sections were incubated overnight with a rabbit polyclonal anti-ACE2 antibody (Santa Cruz Biotechnology Inc), a mouse polyclonal antibody against collagen I (Abcam), a rabbit monoclonal antibody against macrophages (CD68; EMD Millipore, Billerica, MA, USA), and a monoclonal antibody against $\alpha$-smooth muscle actin (SMA, SigmaAldrich Co), respectively. The slides were incubated with the respective antibody $\operatorname{IgG}$ (Vector Laboratories, Burlingame, CA, USA), stained using the ABC-peroxidase kit or ABC-AR (alkaline phosphatase; Vector Laboratories), and substrated with 3,3'-diaminobenzidine tetrahydrochloride or alkaline phosphatase substrate kit (Sigma-Aldrich Co). The quality of immunohistochemistry assay was controlled by either elimination of the primary antibody or incubation with a nonimmune IgG. Sections were counterstained with hematoxylin, dehydrated, and mounted by routine methods. Positive staining was identified as brown and visualized using an Aperio ImageScope CS system (Aperio, Vista, CA, USA). The protein expression levels of ACE2 and collagen I were evaluated by measuring the mean optical density (MOD), and accumulation of macrophages and myofibroblasts was 
calculated as the number of positively stained cells at a high-powered filed.

\section{Evaluation of myocardial fibrosis by Masson's trichrome staining}

Myocardial fibrosis in the tissue sections was determined using Masson's trichrome method and quantitatively analyzed by morphometry as previously reported. ${ }^{4}$ The staining turns collagen blue, nuclei black, and viable muscle fiber red. Eight randomized high-powered fields per tissue section were selected to determine the positively stained collagen areas and microvessels.

\section{Determination of hemodynamics and cardiac performance}

At the end of the experiment, all animals were anesthetized by inhaling $4 \%$ isoflurane and maintained at $2 \%$ isoflurane in oxygen. Both the carotid arteries were cannulated with a polyethylene catheter connected to a Statham transducer to measure the mean carotid pressure via a BL-410 biological signal acquisition and processing system (Techman Software Co, Ltd, Chengdu, China). The polyethylene catheter within the right carotid artery was then inserted into the left ventricle to determine the cardiac performance including heart rate (HR), left ventricular systolic pressure (LVSP), left ventricular end-diastolic pressure (LVEDP), and maximum positive $\left(+\mathrm{dP}_{\mathrm{d}} \mathrm{dt}_{\max }\right)$ and negative $\left(-\mathrm{dP} / \mathrm{dt}_{\max }\right)$ values of the first derivative of left ventricular pressure.

\section{Detection of global cardiac function and ventricular dimension by echocardiography}

A two-dimensional (2D) guided M-mode ultrasound system (Vivid 7; GE Healthcare, Waukesha, WI, USA) was used to assess the left ventricular systolic and diastolic function as previously reported. ${ }^{20}$ In brief, rats were mildly anesthetized with $1.5 \%$ isoflurane, and transthoracic echocardiography was performed using a $15 \mathrm{~s} \mathrm{MHz} \mathrm{linear} \mathrm{transducer.} \mathrm{Percent}$ fraction shortening (FS) and ejection fraction (EF) were calculated. 2D images, which were frozen at the end of diastole, were used to measure left ventricular internal dimension (LVID). All measurements were averaged over three consecutive cardiac cycles.

\section{Statistical analysis}

Data are expressed as mean \pm standard error. A one-way analysis of variance (ANOVA) followed by StudentNewman-Keul's post hoc test was used to analyze group differences in the band intensity of AT1/AT2 receptors, the MOD of ACE2 expression, populations of macrophages and myofibroblasts, and expression of TGF $\beta 1$, collagens, and Smads. Dynamic and echocardiographic data were analyzed by one-way repeated measures ANOVA followed by post hoc analysis with Student-Newman-Keul's test for multiple comparisons using SigmaPlot (Systat Software Inc, San Jose, CA, USA). A $p$-value $<0.05$ was accepted as statistically significant.

\section{Results}

\section{Animal mortality of the study}

Twenty-eight rats were initially included in the present study. Two rats died in the TAC group 3 days after surgery because of excessive stenosis. One rat in the edaravone group and one rat in the telmisartan group were excluded because the pressure gradients between the two carotid arteries were not present in the range of $60-90 \mathrm{mmHg}$. The remaining 24 rats were randomly assigned to the experimental groups, that is, six rats for each group.

\section{Effects of edaravone and telmisartan on oxidative stress, antioxidant enzyme, and cardiac hypertrophy after TAC}

The content of MDA and activity of SOD in the heart were used to estimate the levels of lipid peroxidation and tissue antioxidative ability. As shown in Figure 1A, TAC caused a significant increase in MDA $(22 \% \pm 2 \%)$ and a reduction in SOD $(73 \% \pm 2 \%)$ compared with the Sham group (all $p<0.05$ ), suggesting an enhanced oxidative status after 8 weeks of chronic pressure overload. Relative to the TAC group, administration of edaravone or dietary telmisartan reduced MDA level by $70 \% \pm 5 \%$ and $46 \% \pm 4 \%$, respectively (all $p<0.05$ vs TAC group), and increased SOD enzyme activity by $57 \% \pm 2 \%$ and $53 \% \pm 1 \%$ (all $p<0.05$ vs TAC group), respectively, implying improved antioxidative ability of the heart.

Cardiac hypertrophy was evaluated by calculating the HW/BW ratio and MSA. Eight weeks of chronic elevation of afterload in the left ventricle caused siginificant changes in HW/BW ratio and MSA size in the TAC group. As shown in Figure 1A, the HW/BW ratio in the TAC group increased significantly relative to the Sham group ( $3.2 \pm 0.1$ vs $2.5 \pm 0.1$, $p<0.05)$. Treatment with edaravone or telmisartan comparatively reduced the $\mathrm{HW} / \mathrm{BW}$ ratio relative to the TAC group (2.6 \pm 0.1 in the Edara group and $2.3 \pm 0.2$ in the Telmi group vs $3.2 \pm 0.1$ in the TAC group, all $p<0.05$ ). The inhibitory effect of edaravone or telmisartan on cardiac 

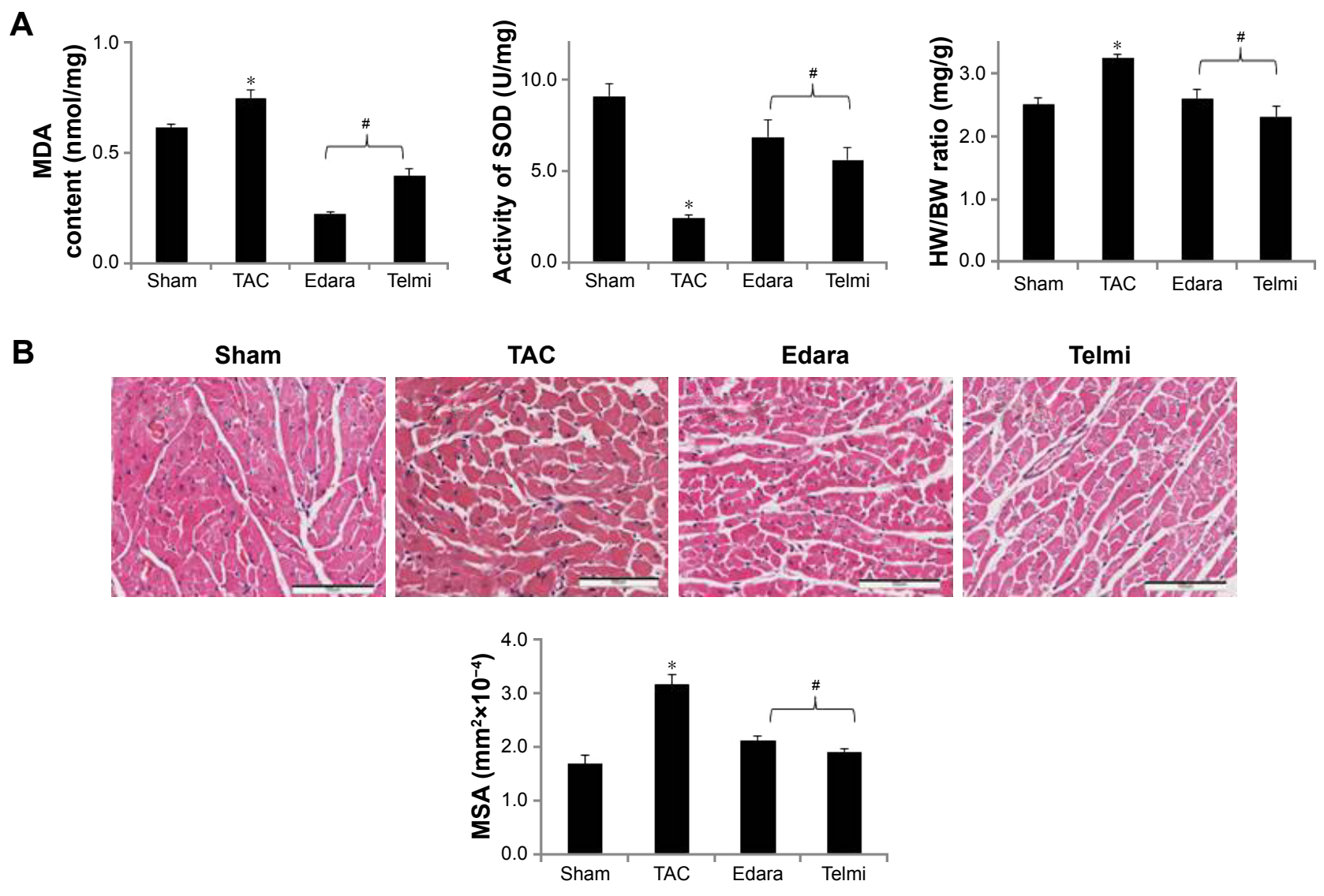

Figure I Lipid peroxidation, antioxidant enzyme activity, and myocardial hypertrophy among the different groups.

Notes: (A) MDA content and SOD activity in myocardial tissue were determined using commercial kits. HW/BW (mg/g) was calculated by weight. (B) MSA in series was measured morphometrically at a HPF (original magnification: $\times 200$; scale bars: $100 \mu \mathrm{m}$ ). Sham: rats underwent the same surgical procedure without banding the aorta; TAC: the ascending aorta was bended for 8 weeks; TAC plus edaravone (Edara): rats received an intraperitoneal injection of edaravone at a dose of $10 \mathrm{mg} / \mathrm{kg} / \mathrm{day}$ for 8 weeks after TAC; TAC plus telmisartan (Telmi): telmisartan was administered via gastric gavage at a dose of $10 \mathrm{mg} / \mathrm{kg} / \mathrm{day}$ for 8 weeks after TAC. Values are mean \pm SEM ( $\mathrm{n}=6 / \mathrm{group}$ ). $* p<0.05$ TAC versus Sham; ${ }^{*} p<0.05$ Edara or Telmi versus TAC.

Abbreviations: MDA, malonaldehyde; SOD, superoxide dismutase; HW/BW, heart to body weight ratio; MSA, myocyte sectional area; HPF, high-powered field; TAC, transverse aortic constriction; SEM, standard error of means.

hypertrophy was further confirmed by a reduced MSA value $\left(2.1 \pm 0.2 \mathrm{~mm}^{2} \times 10^{-4}\right.$ in the Edara group and $1.9 \pm 0.1 \mathrm{~mm}^{2} \times 10^{-4}$ in the Telmi group vs $3.2 \pm 0.1 \mathrm{~mm}^{2} \times 10^{-4}$ in the TAC group, all $p<0.05$; Figure 1B).

\section{Effects of edaravone and telmisartan on expression of Ang II ATI, AT2 receptors, and ACE2 after TAC}

Protein levels of the AT1 and AT2 receptors were analyzed by Western blot assay. The protein of the AT1 receptor in the myocardium was constitutively expressed in the Sham group. Compared with the Sham group, constriction of ascending artery caused a significant increase in the protein level at week 8 (Figure 2A). The intraperitoneal injection of edaravone or oral administration of telmisartan over a 8-week period abrogated the upregulation of the AT1 receptor. The expression of the AT2 receptor protein was downregulated after 8 weeks of TAC. As shown in Figure 2B, the AT2 receptor protein was constitutively presented in the shamoperated rats. However, the level of the AT2 receptor was significantly reduced at week 8 in the TAC group relative to those in the Sham group. The augmentation in protein expression of the AT2 receptor was detected after intraperitoneal injection of edaravone or oral administration of telmisartan. These results suggested that edaravone or telmisartan has dual actions including antagonism of the AT1 receptor and stimulation of the AT2 receptor, evidenced by a decreased ratio of the AT1 receptor over the AT 2 receptor $(0.57 \pm 0.2$ in the edaravone group and $0.54 \pm 0.1$ in the telmisartan group vs $3.1 \pm 0.2$ in the TAC group, all $p<0.05$ ).

ACE2 functions as a regulator to oppose the AT1 receptormediated vasoconstriction, hypertrophy, and fibrosis. ${ }^{12}$ In the present study, the expression of ACE2 in myocardial tissue was examined by immunohistochemistry. It is clearly shown that the presence of pressure overload significantly alters the expression of ACE2. MOD of ACE2 was significantly 
A
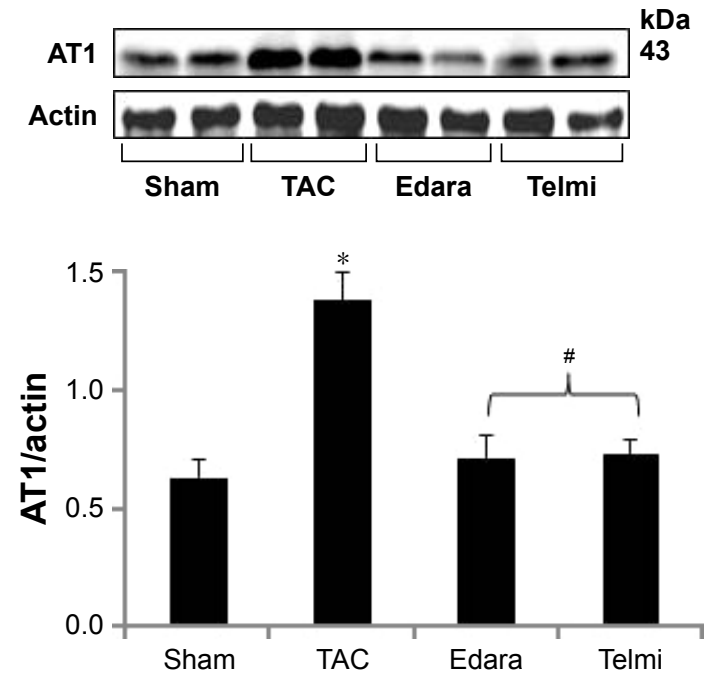

C

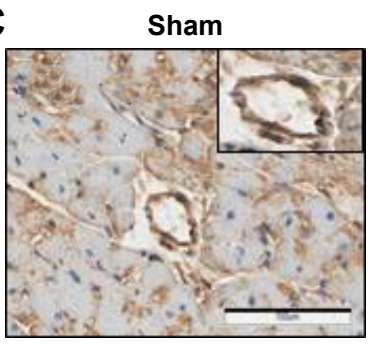

TAC

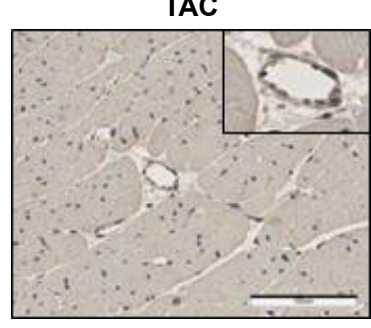

B
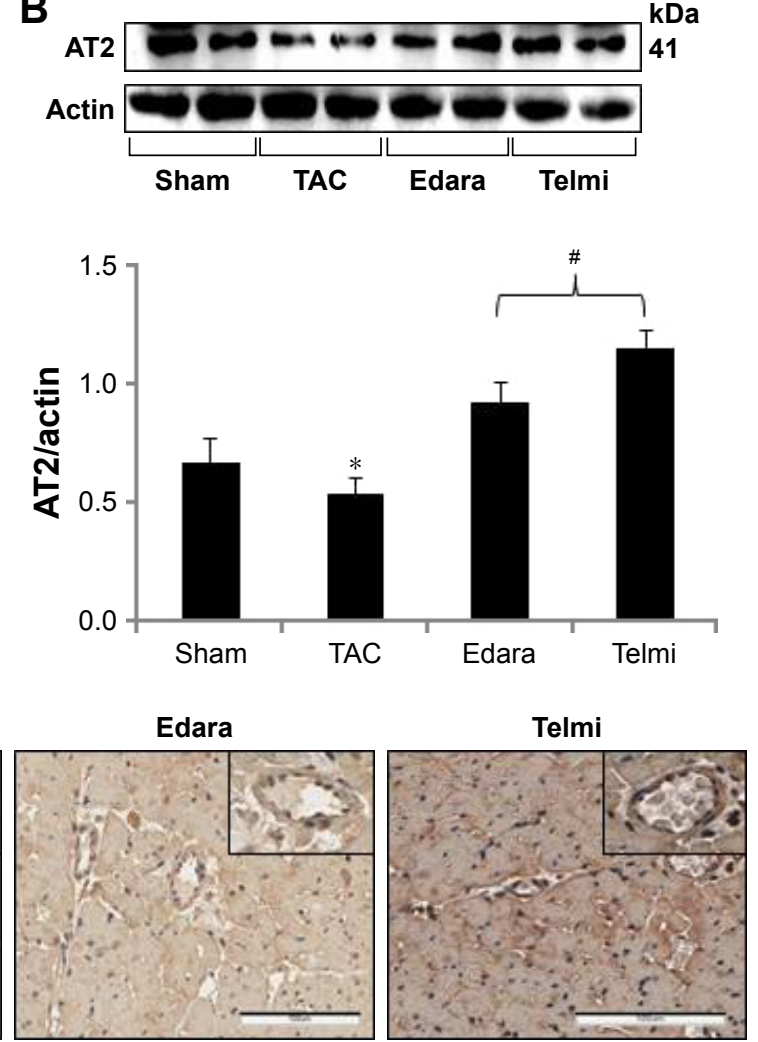

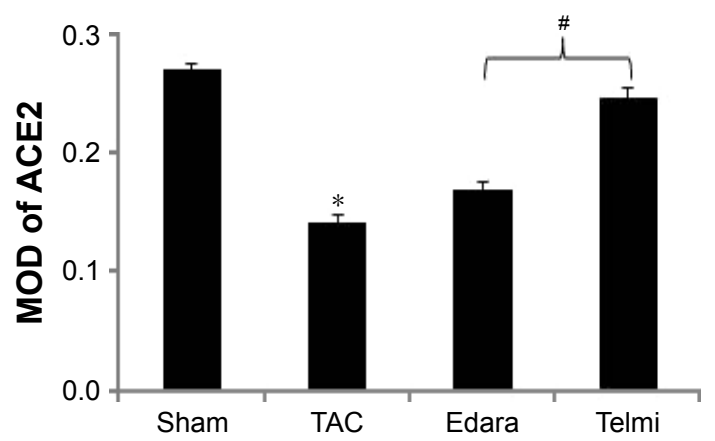

Figure 2 Expression of Ang II ATI/AT2 receptors and ACE2 in the myocardium.

Notes: (A, B) Protein level of ATI and AT2 receptors detected by Western blot. All bands were normalized by actin as illustrated in the bar graphs. ACE2 in the intermyocardium and intracardiac vessels (insets) was determined using immunohistochemical staining $(\mathbf{C})$ and calculated as MOD in the tissue section. Original magnification: $\times 200$; scale bars: $100 \mu \mathrm{m}$. Values are mean \pm SEM ( $n=6 /$ group). ${ }^{*} p<0.05$ TAC versus Sham; ${ }^{p} p<0.05$ Edara or Telmi versus TAC.

Abbreviations: MOD, mean optical density; ACE2, angiotensin converting enzyme 2; Ang II, angiotensin II; SEM, standard error of means; TAC, transverse aortic constriction.

reduced in the myocardium relative to the Sham group at week 8 (Figure 2C). The reduced expression of ACE2 in the intracardiac vessels and myocardium was reversed with intraperitoneal injection of edaravone or by oral administration of telmisartan during 8 weeks of TAC.

\section{Effects of edaravone and telmisartan on infiltration of macrophages, expression of TGF $\beta$ I, and proliferation of myofibroblasts after TAC}

It is known that TGF $\beta 1$ released from accumulated monocytederived macrophages in the heart is involved in proliferation and differentiation of fibroblasts to myofibroblasts. ${ }^{6}$ In the present experiment, macrophage infiltration and myofibroblast proliferation in the myocardium were detected using immunohistochemical staining. Few macrophages are detected in the Sham group. TAC caused a significant increase in the number of positively stained macrophages in the intravascular region and interstitial myocardium (Figure 3A). The time course in macrophage migration was consistent with the upregulated AT1 receptor (Figure 2A). The expression level of TGF $\beta 1$ protein was detected by Western blot analysis. As shown in Figure 3B, TGF $\beta 1$ was constitutively expressed in the Sham group. After 8 weeks 
A

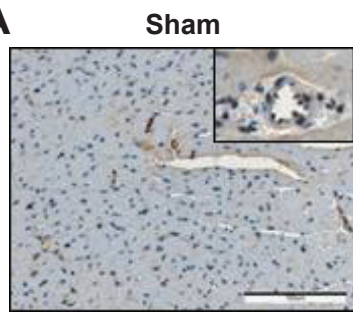

Edara

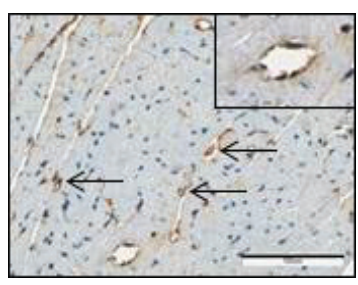

B

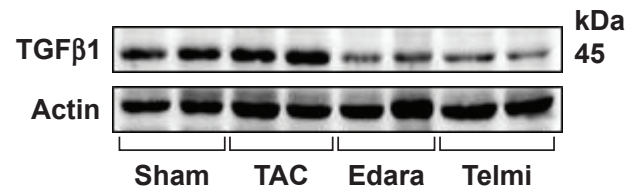

C

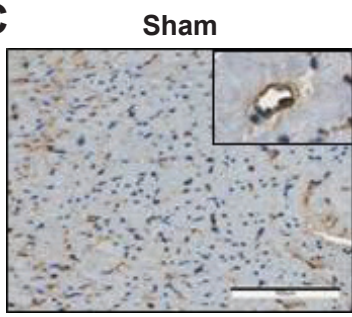

Edara

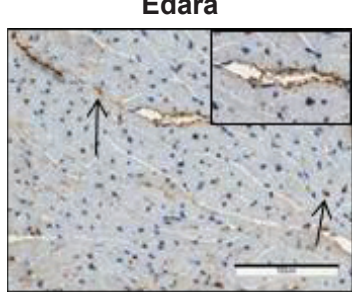

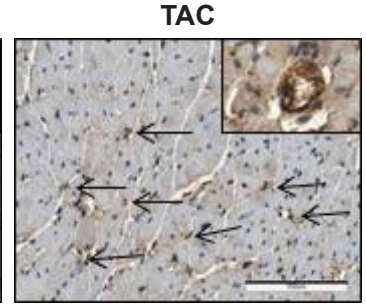

Telmi

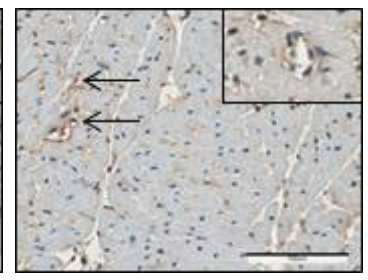

$\mathrm{kDa}$ 45

TAC

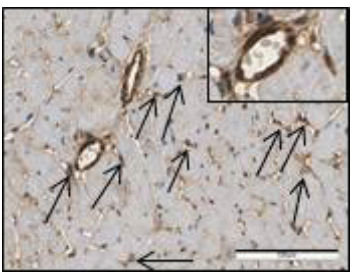

Telmi

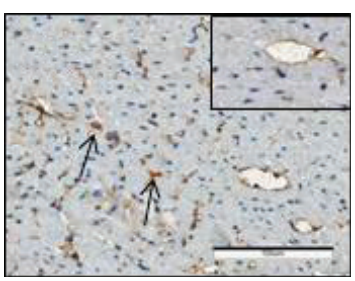

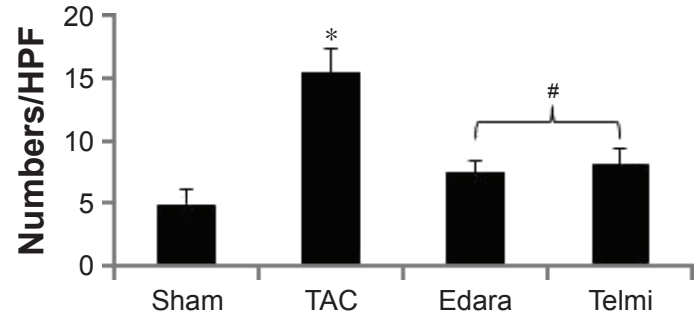
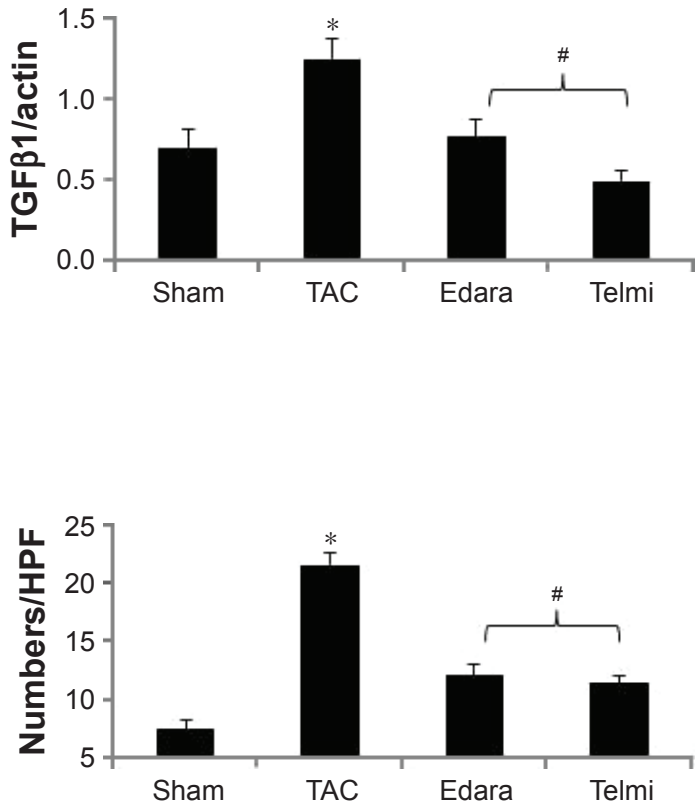

Figure 3 Macrophage accumulation, TGF $\beta$ I protein expression, and myofibroblast proliferation among the different groups.

Notes: (A) Accumulation of macrophages and (C) proliferation of myofibroblasts were determined by the number of positively stained cells (bar graphs) using immunohistochemical staining at each HPF (magnification: $\times 200$, scale bars: $100 \mu \mathrm{m}$ ), as indicated by the arrows. (B) Representative of TGF $\beta$ I protein expression analyzed by Western blot. All bands were normalized by actin, respectively. Values are mean \pm SEM (n=6/group). ${ }^{*}<0.05$ TAC versus Sham; ${ }^{*} p<0.05$ Edara or Telmi versus TAC. Abbreviations: HPF, high-powered field; TAC, transverse aortic constriction; SEM, standard error of means; TGF $\beta$ I, transforming growth factor beta I.

of TAC, TGF $\beta 1$ protein level was significantly upregulated relative to the Sham group. Consistent with the accumulation of macrophages, positively stained $\alpha$-SMA-expressing myofibroblasts, an indicater of fibroblast proliferation, were significantly increased by TAC at week 8 . As shown in Figure 3C, few $\alpha$-SMA positive cells are seen in the vascular smooth muscle and myocardium in the Sham group. However, the number of positively stained $\alpha$-SMA myofibroblasts, which are aligned with the host myocardial fibers, significantly increased after 8 weeks of TAC. Administration of edaravone or telmisartan during TAC comparatively reduced the number of accumulated macrophages, expression of TGF $\beta 1$, and proliferation of myofibroblasts at week 8 compared with the TAC group (Figure 3).

\section{Effects of edaravone and telmisartan on expression of Smads after TAC}

Proteins of the Smad family mediate signal transduction of the TGF $\beta$, and are associated with regulation of collagen synthesis following myofibroblast proliferation. Overexpression of 
Smad2, Smad3, or Smad4 protein promotes the production of collagens, but Smad7 negatively regulates TGF $\beta$-mediated signaling. ${ }^{4}$ In the present study, the protein level of the Smad family was evaluated by Western blot assay. As shown in Figure 4, the protein levels of Smad2 and Smad3 were increased significantly at week 8 after TAC relative to the Sham control, in coincidence with the proliferation of myofibroblasts. The expression of Smad4 was not altered at the end of 8 weeks of TAC in all groups, but the level of Smad7 was significantly downregulated at this time point relative to the Sham group. Administration of edaravone or telmisartan comparatively reduced protein levels of $\mathrm{Smad} 2 / 3$ and enhanced the level of Smad7 compared with the TAC group (Figure 4).
Effects of edaravone and telmisartan on synthesis of collagens and formation of fibrotic tissue after TAC

Excess collagen deposition and fibrosis have been linked to myocardial stiffness, and cardiac diastolic and systolic dysfunction. ${ }^{4}$ In the present study, expression of collagen I, collagen III, and fibrotic tissue formation were analyzed using immunohistochemistry, Western blot assay, and Masson's trichrome staining. As shown in Figure 5A, collagen I was barely detected in the Sham group. After 8 weeks of TAC, the deposition of collagen I not only occurred in the matrix of vascular endothelium, but also was excessively expressed in the myocardium. Treatment
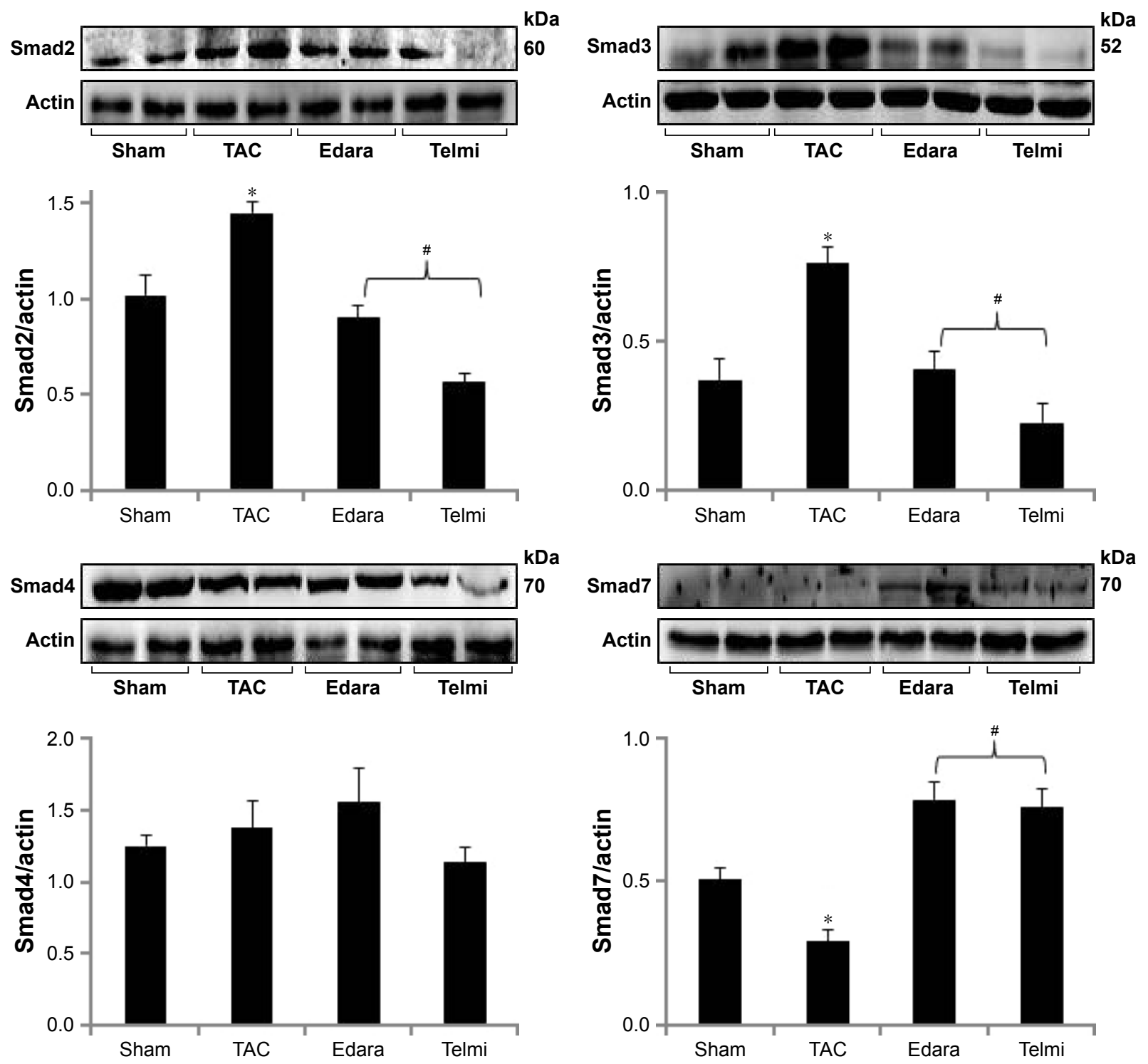

Figure 4 Protein expression levels of Smad2, Smad3, Smad4, and Smad7 among the different groups.

Notes: Protein expression levels of different Smads subtypes were analyzed using Western blot. All bands were normalized by actin among groups as illustrated in the bar graphs. Values are mean \pm SEM ( $n=6 /$ group). ${ }^{*} p<0.05$ TAC versus Sham; ${ }^{*} p<0.05$ Edara or Telmi versus TAC.

Abbreviations: TAC, transverse aortic constriction; SEM, standard error of means. 
A

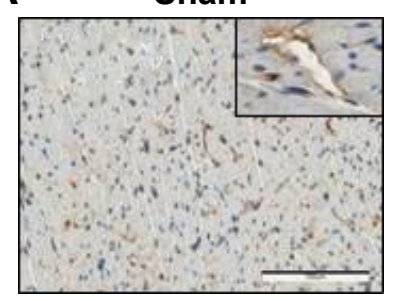

Edara

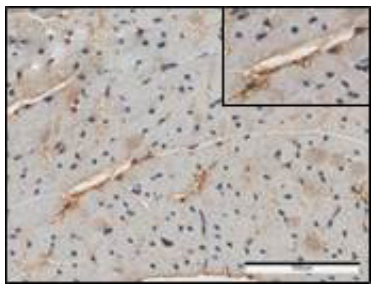

B

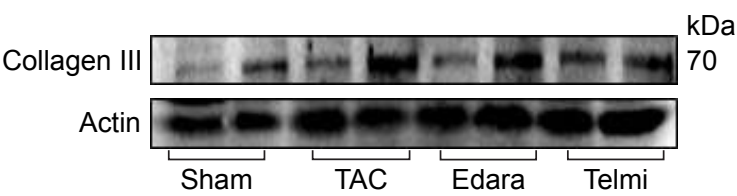

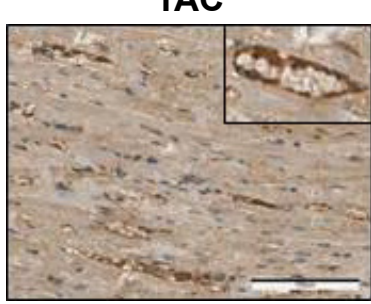

Telmi

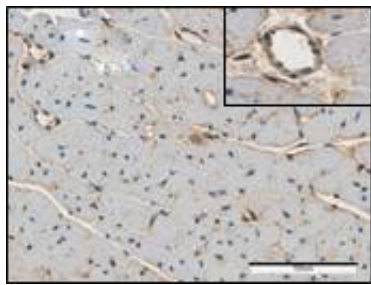

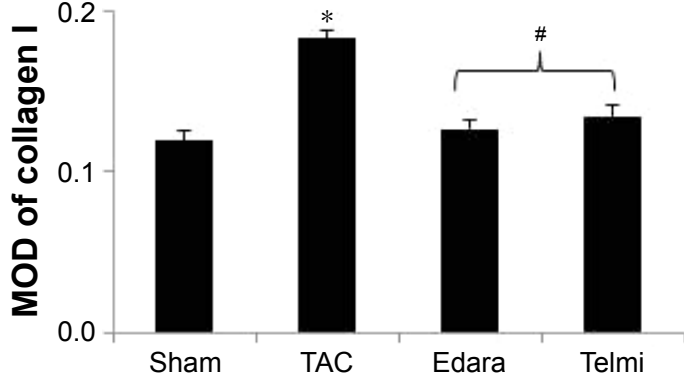

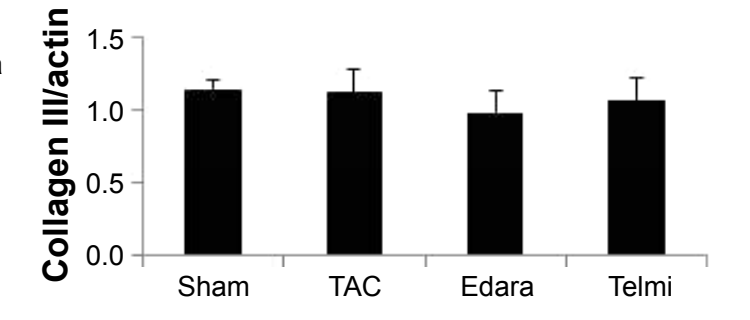

C
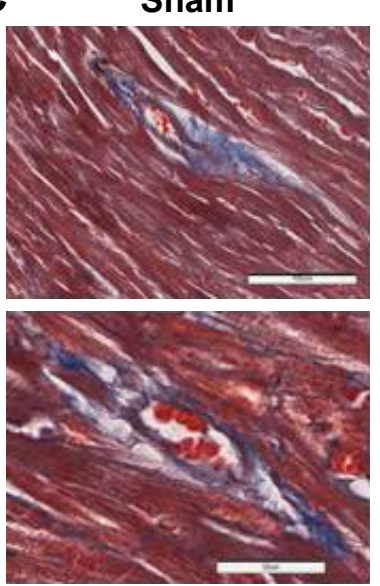

TAC
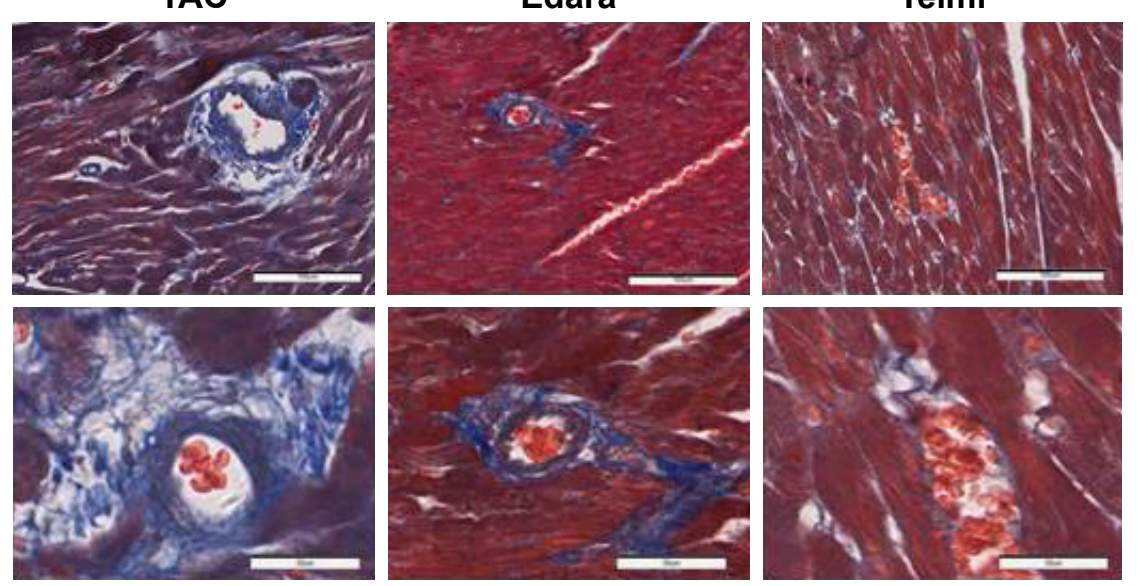

Figure 5 Detection of collagen type I, III, and fibrosis among the different groups.

Notes: (A) Deposition of collagen I in the myocardium and blood vessels (inset) was detected by immunohistochemical staining and calculated by MOD. (B) Protein expression level of collagen III was analyzed by Western blot. All bands were normalized by actin as illustrated in the bar graphs. (C) Interstitial and perivascular fibrosis in the myocardial sections was identified as blue collagen staining using Masson's trichrome method. All images were viewed under magnification: $\times 200$ (scale bars: $100 \mu m$ ). Values are mean \pm SEM ( $n=6$ /group). ${ }^{*} p<0.05$ TAC versus Sham; ${ }^{*} p<0.05$ Edara or Telmi versus TAC.

Abbreviations: MOD, mean optical density; TAC, transverse aortic constriction; SEM, standard error of means.

of edaravone or telmisartan comparatively reduced deposition of collagen I relative to the TAC group. There was no significant difference in the expression of collagen III protein among all groups (Figure 5B). Consistent with the increased synthesis of collagen I, the production of fibrotic tissue in the perivascular area and interstitial myocardium as identified by Masson's trichrome staining was significantly augmented by TAC. However, more organized and circumscribed fibers in the myocardium were detected in edaravone or telmisartan-treated rats after 8 weeks of TAC (Figure 5C), indicating less fibrosis formation at interstitial and perivascular regions.

\section{Effects of edaravone and telmisartan on hemodynamics and cardiac performance after TAC}

To control the degree of aortic stenosis, blood pressure in the left and right carotid arteries was measured. Animals with a 
trans-stenosal pressure difference between 60 and $90 \mathrm{mmHg}$ were included in the present study. There were no group differences in HR during the 8-week period after TAC as shown in Figure 6A. The left mean carotid pressure was relatively stable during the 8-week period after TAC (data not shown) among all groups. As shown in Figure 6B, TAC caused a significant reduction in LVSP $(110 \pm 8$ vs $151 \pm 8 \mathrm{mmHg}$ in Sham, $p<0.05)$ and the maximum rate of $+\mathrm{dp} / \mathrm{dt}_{\max }(4.4 \pm 0.6$ vs $8.4 \pm 0.5 \mathrm{mmHg} / \mathrm{s} \times 10^{3}$ in Sham, $p<0.05$ ) after 8 weeks of TAC. These alterations were significantly reversed with treatment of edaravone or telmisartan, suggesting beneficial effects of both drugs on cardiac systolic performance.

LVEDP, as the representative of left ventricular end-diastolic circumference, was significantly increased at week 8 (13 \pm 2 vs $7 \pm 1 \mathrm{mmHg}$ in Sham, $p<0.05$; Figure $6 \mathrm{C}$ ). This was consistent with the reduced maximum rate of $-\mathrm{dp} / \mathrm{dt}_{\text {max }}(3.8 \pm 0.5 \mathrm{vs}$ $7.5 \pm 0.5 \mathrm{mmHg} / \mathrm{s} \times 10^{3}$ in Sham, $p<0.05$; Figure 6C), indicating impaired isovolumetric relaxation. Treatment with edaravone or telmisartan significantly decreased LVEDP $(3 \pm 1 \mathrm{mmHg}$ in the Edara group and $7 \pm 1 \mathrm{mmHg}$ in the Telmi group vs $13 \pm 2 \mathrm{mmHg}$ in the TAC group, all $p<0.05)$, and increased the maximum rate of $-\mathrm{dp} / \mathrm{dt}_{\text {max }}\left(10.8 \pm 1.5 \mathrm{mmHg} / \mathrm{s} \times 10^{3}\right.$ in the Edara group and $6.4 \pm 0.6 \mathrm{mmHg} / \mathrm{s} \times 10^{3}$ in the Telmi group vs $3.8 \pm 0.5 \mathrm{mmHg} / \mathrm{s} \times 10^{3}$ in the TAC group, all $p<0.05)$, suggesting less dilatation in the ventricular chamber (Figure 6C).

A

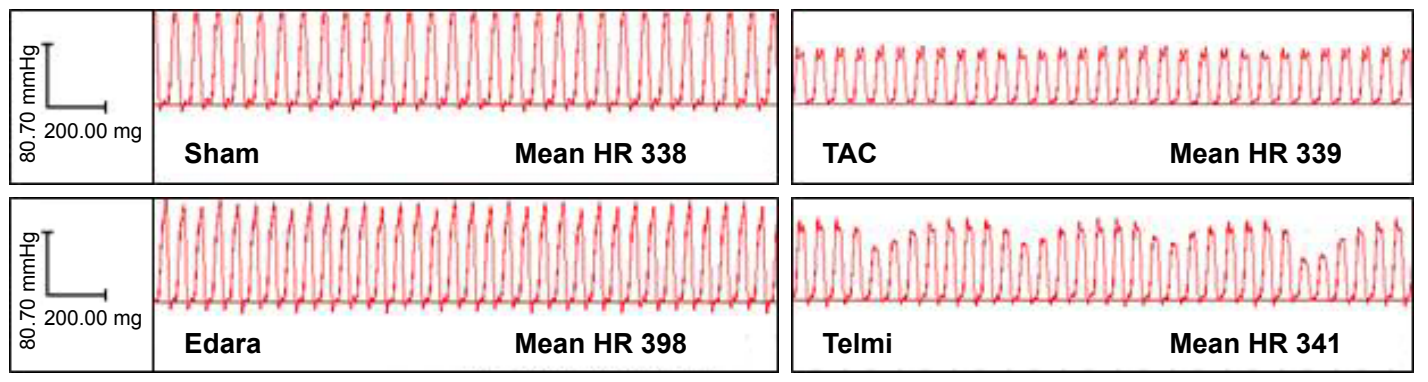

B
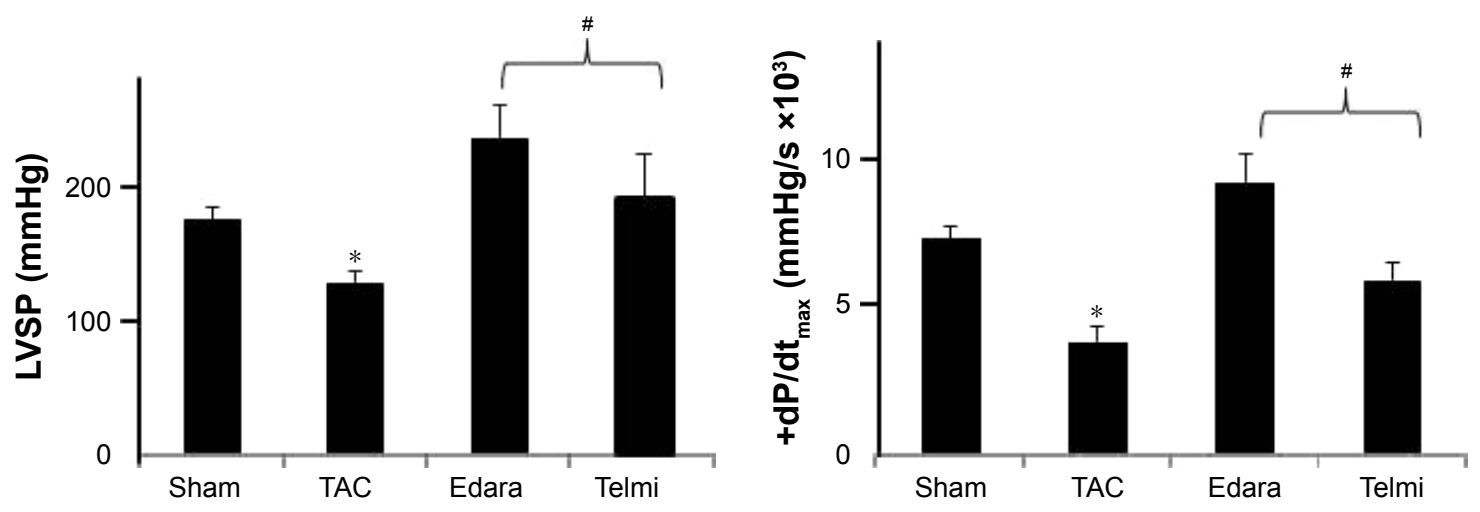

C
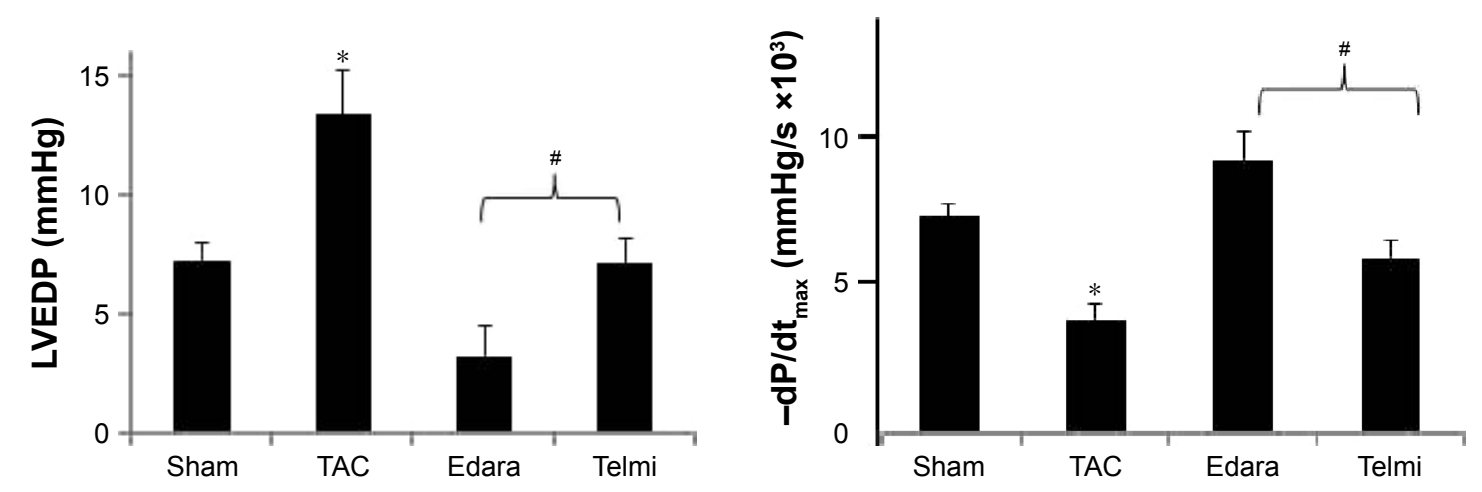

Figure 6 Heart rate and left ventricular performance.

Notes: (A) Representative recording of HR and left ventricular pressure among the different groups. Bar graphs show changes in LVSP and LVEDP (B) and changes in maximum positive $\left(+d P / d t_{\max }\right)$ and negative $\left(-d P / d t_{\max }\right)$ values of the first derivative of left ventricular pressure $(\mathbf{C})$. Values are mean $\pm S E M(n=6 / g r o u p)$. ${ }^{*} p<0.05 T A C$ versus Sham; ${ }^{*} p<0.05$ Edara or Telmi versus TAC.

Abbreviations: LVEDP, left ventricular end-diastolic pressure; LVSP, left ventricular systolic pressure; TAC, transverse aortic constriction; SEM, standard error of means; $\mathrm{HR}$, heart rate. 
A

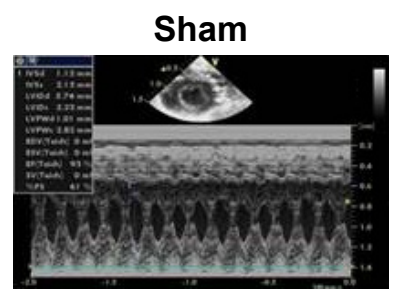

B

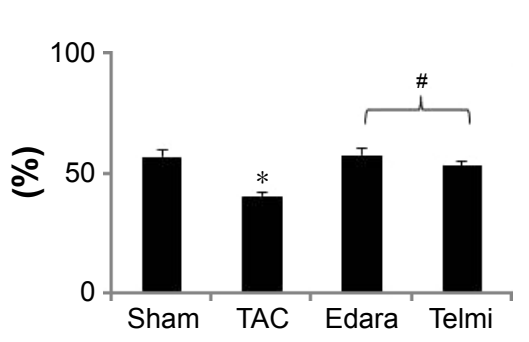

TAC

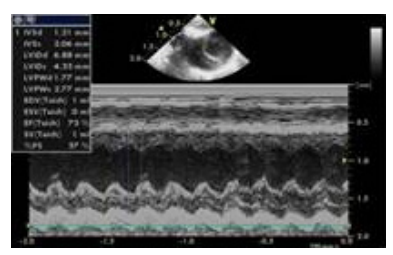

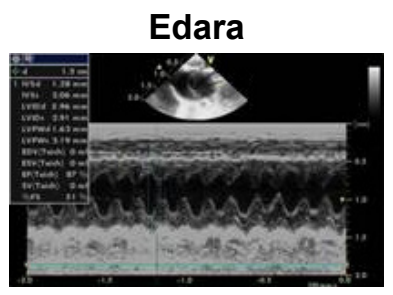

$\mathrm{EF}$
Telmi

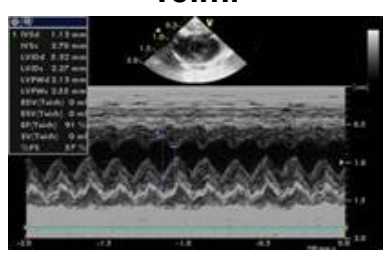

LVID
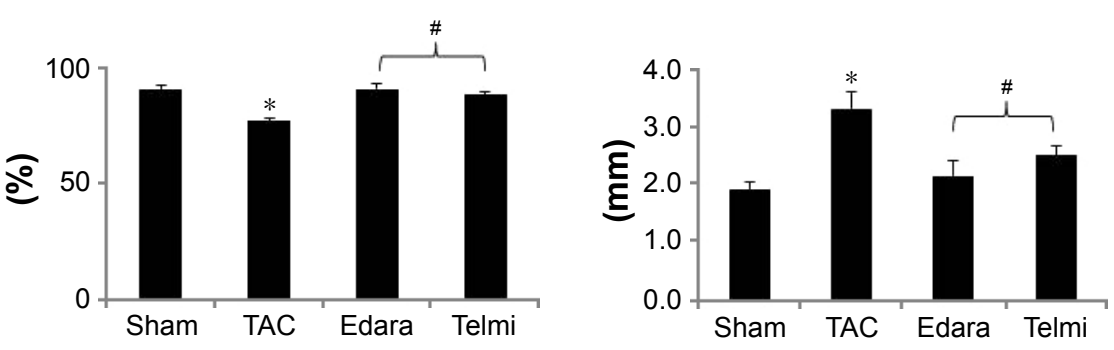

Figure 7 Echocardiographic assessment of left ventricular systolic function among the different groups.

Notes: (A) Two-dimensional echocardiographic images represent cardiac global function. (B) Cardiac systolic function was determined by measuring FS and EF. LVID was measured at end diastole. Values are mean \pm SEM ( $n=6 /$ group). ${ }^{*} p<0.05$ TAC versus Sham; ${ }^{*}<0.05$ Edara or Telmi versus TAC.

Abbreviations: LVID, left ventricular internal dimension; FS, fraction shortening; EF, ejection fraction; TAC, transverse aortic constriction; SEM, standard error of means.

\section{Effects of edaravone and telmisartan on global cardiac function and left ventricular dimension}

The fibrotic change in the pressure overloaded heart was associated with alterations in chamber dimension and progressive impairment of the left ventricular function as measured by echocardiography (Figure 7A). After 8 weeks of TAC, the indexes of systolic function including FS and EF were significantly impaired relative to those in the sham-operated hearts (Figure 7B), implying systolic dysfunction. Administration of edaravone or telmisartan for 8 weeks during TAC significantly improved the contractile function relative to TAC alone, with an increased $\mathrm{EF}$ in the Edara group $(75 \% \pm 2 \%)$ and in the Telmi group $(73 \% \pm 1 \%$; all $p<0.05$ ) versus the TAC group. These alterations were consistent with a change in LVID at the end of the diastole. As shown in Figure 7B, LVID increased significantly at week 8 after TAC, but was effectively preserved by treatment with edaravone or telmisartan. These results were in accordance with the reduced LVEDP as measured directly from the heart.

\section{Discussion}

In this study, treatment with edaravone reduced the content of MDA and increased the activity of SOD. Enhanced expression of the AT1 receptor by TAC was downregulated, and AT2 receptor and ACE2 were upregulated. Along with these modifications, migration of macrophages and proliferation of myofibroblasts were inhibited and TGF $\beta 1 /$ Smads-mediated fibrotic signaling pathways were attenuated, which was accompanied by a significant improvement in cardiac function. Comparative inhibition between edaravone and telmisartan on TAC-induced deleterious changes suggested that protection with edaravone is potentially mediated by inhibiting AT1 receptor-evoked fibrotic signaling.

The rat model of TAC to simulate aortic stenosis in humans was chosen for the present study. This chronic increase in workload is independent of blood pressure alteration, leading to pathological hypertrophy of the heart. During early stages of TAC (usually $<4$ weeks), it is characterized by compensatory concentric hypertrophy with diastolic dysfunction, whereas at the later stages, ventricular dilatation occurs with development of eccentric hypertrophy and systolic dysfunction. Eventually, these maladaptive responses to the chronic hemodynamic overload result in heart failure. ${ }^{21}$ The time course in the present study lasted 8 weeks. Data clearly showed that significant ventricular dilatation and systolic dysfunction are identified at this time point. We may miss the time window of showing concentric hypertrophy and diastolic dysfunction with TAC at early stages. However, treatment with edaravone for 8 weeks significantly reduced chamber dilatation and attenuated systolic dysfunction, suggesting its role in preventing progression of cardiac dysfunction. It is worth to further check the time course in edaravone-exerted cardioprotection, and whether early attenuation of concentric hypertrophy with 
treatment of edaravone prevents development of ventricular dilatation and dysfunction.

It is well known that the majority of deleterious effects by Ang II are mediated via the AT1 receptor, whereas the AT2 receptor is suggested to counter-regulate the action of the AT1 receptor. ${ }^{8}$ In the present study, we were able to demonstrate the reciprocal expression between AT1 and AT2 receptor after TAC, as evidenced by upregulated AT1 receptor, downregulated AT2, and elevated AT1/AT2 ratio. It has been demonstrated that induction of ROS is associated with Ang II stimulation. In the rat model of Ang II infusion, activation of the AT1 receptor causes excessive production of ROS evidenced by an increased end product of lipid peroxidation (MDA) and attenuated antioxidant enzyme (SOD). ${ }^{22,23}$ In contrast, stimulation of the AT2 receptor downregulates activity of NADPH oxidase and reduces NOX-derived ROS production. ${ }^{24,25}$ The antioxidant action of edaravone has been previously linked to attenuation of pressure overload-induced left ventricular hypertrophy and the progression of dilated cardiomyopathy. ${ }^{17,18}$ In the present study, we demonstrated that in coincidence with inhibition of the AT1 receptor and upregulation of the AT2 receptor, edaravone reduces MDA content and increases SOD activity. Comparative modulation in MDA and SOD between edaravone and telmisartan suggested that inhibition of oxidant production with edaravone can be potentially achieved by inhibiting the AT1 receptor. However, we need to further demonstrate alternative signaling pathways involved in inhibition of the AT1 receptor in both cardiomyocytes and vascular smooth muscle cells with edaravone. In this regard, the downregulation of the AT1 receptor with the AT1 blockade and antioxidant has been associated with molecular modulations of several complex signaling proteins and transcription factors such as peroxisome proliferator-activated receptor $\gamma$, ERK1/2, P38 MAPK, Pyk2, c-Src family kinases, $\mathrm{NF \kappa B}$, and JAK/STAT. ${ }^{25,26}$

ACE2 is a homolog of ACE and converts Ang II to Ang (1-7), which is responsible for vasodilation and antagonism to reduce proliferation/fibrosis. ${ }^{27}$ ACE2 can be detected on vascular endothelium, smooth muscle cells, and cardiomyocytes. ${ }^{28}$ In the present study, ACE2 expression was examined from the paraffin embedded tissue blocks using immunohistochemical staining. Downregulation of ACE2 expression in the blood vessels and myocardium by TAC may further enhance AT1 receptor signaling. Consistent with the action of the AT1 receptor antagonism, treatment with edaravone or telmisartan for 8 weeks significantly increased ACE2 expression. These data suggested that signaling in inhibition of cardiac fibrosis evoked by edaravone or telmisartan includes the dual action of AT1 receptor inhibition and ACE2 activation.

Accumulating evidence has indicated that infiltration of macrophages to the myocardium is involved in the development of vascular and myocardial fibrosis. ${ }^{29,30}$ Activation of the AT1 receptor on macrophages releases proinflammatory cytokines, such as TGF $\beta 1$, tumor necrosis factor- $\alpha$, inducible nitric oxide synthases, interleukin- $1 \beta$ and IL-6, resulting in transdifferentiation of fibroblasts to myofibroblasts. ${ }^{31}$ Accordingly, inhibition of TGF $\beta 1$ expression suppresses fibroblast proliferation and myocardial fibrosis. ${ }^{32}$ In the present study, we found that along with the reduction in infiltration of macrophages by edaravone, expression of TGF $\beta 1$ was attenuated and proliferation of myofibroblasts was inhibited, indicating that macrophages function as a master regulator of myofibroblasts. A comparable reduction in the level of upregulated TGF $\beta 1$ expression with edaravone and telmisartan further suggested that these processes are mediated by inhibiting the AT1 receptor. These data were consistent with previous studies showing that treatment with edaravone reduces the expression of TGF $\beta 1$ and collagens in rats exhibiting dilated cardiomyopathy ${ }^{18}$ and attenuates cardiac fibrosis induced by TAC in mice. ${ }^{17}$

TGF $\beta 1$ released from macrophages promotes differentiation of myofibroblasts and then stimulates phosphorylation of Smad2/3 to form a heterotrimeric complex with Smad4, subsequently enhancing expression of collagen genes in the nucleus. ${ }^{33,34} \mathrm{Smad} 7$ is an inhibitor of signaling by preventing the phosphorylation of Smad2/3 and interfering in the formation of Smads complex. ${ }^{35}$ In the present study, treatment with edaravone inhibited the expression of Smad2/3, upregulated Smad7, and reduced the synthesis of collagen I to comparable levels as seen in telmisartan-treated animals. In accordance with these modifications, immunohistochemistry and Masson's staining also revealed that deposition of collagens and formation of fibrosis are reduced in the perivascular area and intermyocardium. These data suggested that signaling pathways involved in the attenuation of cardiac fibrosis by edaravone are associated with the inhibition of the AT1 receptor expression.

Compensatory hypertrophy with impaired left ventricular diastolic function but preserved EF can be detected as early as 2 weeks after aortic constriction, whereas maladaptive left ventricular dilatation with declined cardiac systolic function gradually occurs after 4 weeks. ${ }^{36,37}$ It has been reported that increases in myocyte lengthening from series sarcomere formation and ventricular chamber dimension are attributed to 
the eccentric hypertrophy and progression to failure. ${ }^{38}$ In mice, attenuation of cardiac concentric hypertrophy with treatment of edaravone after 7 days of TAC has been previously reported. ${ }^{17}$ In the present study, we selected an 8-week experimental period and aimed to observe the effect of edaravone on the morphological change and progression of cardiac dysfunction after TAC. Our data showed that changes in the heart/body weight ratio, LVID, and MSA in series suggested the development of eccentric hypertrophy. It is clearly established in the present study that LVSP, $\mathrm{dp} / \mathrm{dt}_{\max }$, and EF are reduced and LVEDP is increased, suggesting cardiac systolic dysfunction. One mechanism involved in alteration of LVEDP is associated with a decrease in ventricular compliance and elevated filling pressure, often attributes to increasing fibrosis. ${ }^{39,40}$

It is known that stimulation of the AT1 receptor has minimal direct inotropic effects on the heart and preservation of cardiac diatolic/systolic function with blockade of the AT1 receptor is primarily mediated by reducing proinflammatary cytokines, tissue hypertrophy, and fibrosis. ${ }^{41}$ In the present study, along with a significant reduction in lipid peroxidation, collagen deposition, and fibrosis with treatment of edaravone or telmisartan, cardiac systolic function was improved, further indicating that pressure overload-induced tissue fibrosis is associated with the progression of cardiac dysfunction.

TAC induces aortic stenosis by narrowing aortic lumen without producing significant effects on hemodynamics (ie, blood pressure) and therefore creates a direct effect on myocardial morphology. In this regard, it has been previously reported that edaravone has no significant effect on vascular tone. In the present study, we found that the left mean carotid pressure is stable during the 8 -week period after TAC with treatment of edaravone (data not shown), indicating that the preservation of cardiac function with edaravone is largely mediated through its direct effect on the heart via inhibiting AT1 receptor-mediated events. Furthermore, comparative protection in cardiac function between edaravone and telmisartan also suggested that anticardiac fibrosis by inhibiting the AT1 receptor can be achieved without significant effect on blood pressure.

\section{Study limitation}

Firstly, Ang II binds to and activates AT1 and AT2 receptors. In the present study, the reciprocal expression between AT1 and AT2 receptor after TAC is identified, that is, upregulated AT1 receptor and downregulated AT2 receptor. Treatment with edaravone has a dual effect on inhibition of the AT1 receptor and activation of the AT2 receptor.
It has been reported that the AT2 receptor antagonizes AT1 receptor-mediated responses by direct binding to the AT1 receptor. This switching between these two receptors is an area of current interest. ${ }^{42}$ Therefore, future study is needed to illustrate whether edaravone can affect the functional receptor numbers (ie, plasma membrane densities) of AT1 and AT2 receptors via ligand binding. Secondly, it has been well demonstrated that aldosterone released from the adrenal gland via the AT1 receptor not only induces tissue fibrosis, but also impairs vascular smooth muscle cell function. ${ }^{43}$ High level of plasma aldosterone in patients with congestive heart failure and hypertension is also detected. ${ }^{44}$ Therefore, more research is needed to better understand the link of potential protection between vascular smooth muscle cells and cardiomyocytes by targeting aldosterone-mediated signaling with edaravone. Finally, there is increased evidence suggesting synergism between Ang II and aldosterone, making the addition of an aldosterone receptor antagonist to the standard therapies of ACEi or angiotensin receptor blocker a strong consideration. ${ }^{45}$ Therefore, it will be necessary to test whether there is an additive effect of edaravone with these drugs on hypertension and heart failure.

\section{Conclusion}

Taken together, we demonstrate that treatment with edaravone inhibits cardiac fibrosis and improves left ventricular function in the setting of 8 weeks of pressure overload in rats after TAC. Mechanisms of action underlying prevention of myocardial fibrosis and improvement of cardiac systolic function may associate with an inhibition of Ang II AT1 receptormediated signaling pathways. Given the fact that there is no unique therapy in the treatment of heart failure, edaravone, as an antioxidant compound, might be used in combination with $\beta$-blocker, ACEi, or Ang II receptor blocker in the management of patients with fibrosis-derived heart failure to prevent the progression of cardiac dysfunction.

\section{Acknowledgments}

This study was supported in part by grants from the Mercer University School of Medicine, the Medcen Community Health Foundation, Georgia, and the National Natural Science Foundation of China (81470436).

\section{Disclosure}

The authors report no conflicts of interest in this work.

\section{References}

1. Travers JG, Kamal FA, Robbins J, Yutzey KE, Blaxall BC. Cardiac fibrosis: the fibroblast awakens. Circ Res. 2016;118(6):1021-1040. 
2. Hill JA, Olson EN. Cardiac plasticity. N Engl J Med. 2008;358(13): 1370-1380.

3. Krenning G, Zeisberg EM, Kalluri R. The origin of fibroblasts and mechanism of cardiac fibrosis. J Cell Physiol. 2010;225(3):631-637.

4. Bai F, Pang XF, Zhang LH, et al. Angiotensin II AT1 receptor alters ACE2 activity, eNOS expression and CD44-hyaluronan interaction in rats with hypertension and myocardial fibrosis. Life Sci. 2016;153: 141-152.

5. Crowley MJ, Powers BJ, Myers ER, McBroom AJ, Sanders G. Angiotensin converting enzyme inhibitors and angiotensin II receptor blockers for treatment of ischemic heart disease: future research needs prioritization. Am Heart J. 2012;163(5):777-782.

6. Zhang LH, Pang XF, Bai F, et al. Preservation of glucagon-like peptide-1 level attenuates angiotensin II-induced tissue fibrosis by altering AT1/ AT2 receptor expression and angiotensin-converting enzyme 2 activity in rat heart. Cardiovasc Drugs Ther. 2015;29(3):243-255.

7. Jones ES, Vinh A, McCarthy CA, Gaspari TA, Widdop RE. AT receptors: functional relevance in cardiovascular disease. Pharmacol Ther. 2008;120(3):292-316.

8. Namsolleck P, Recarti C, Foulquier S, Steckelings UM, Unger T. AT 2 receptor and tissue injury: therapeutic implications. Curr Hypertens Res. 2014;16(2):416-426.

9. Bhaskar Rao A, Prasad E, Deepthi SS, et al. Wound healing: a new perspective on glucosylated tetrahydrocurcumin. Drug Des Devel Ther. 2015;9:3579-3588.

10. Patel VB, Bodiga S, Basu R, et al. Loss of angiotensin-converting enzyme-2 exacerbates diabetic cardiovascular complications and leads to systolic and vascular dysfunction: a critical role of the angiotensin II/ AT1 receptor axis. Circ Res. 2012;110(10):1322-1335.

11. Koka V, Huang XR, Chung A, Wang WS, Truong LD, Lan HY. Angiotensin II up-regulates angiotensin I converting enzyme (ACE), but down-regulates ACE2 via the AT1-ERK/p38 MAP kinase pathway. Am J Pathol. 2008;172(5):1174-1183.

12. Bangalore S, Fakheri R, Toklu B, Ogedegbe G, Weintraub H, Messerli FH. Angiotensin-converting enzyme inhibitors or angiotensin receptor blockers in patients without heart failure? Insights from 254,301 patients from randomized trials. Mayo Clin Proc. 2016;91(1):51-60.

13. Ram CVS. Angiotensin receptor blockers: current status and future prospects. Am J Med. 2008;121(8):656-663.

14. Kikuchi K, Tancharoen S, Takeshige N, et al. The efficacy of edaravone (radicut), a free radical scavenger, for cardiovascular disease. Int $\mathrm{J} \mathrm{Mol}$ Sci. 2013;14(7):13909-13930.

15. Lapchak PA. A critical assessment of edaravone acute ischemic stroke efficacy trials: is edaravone an effective neuroprotective therapy? Expert Opin Pharmacother. 2010;11(10):1753-1763.

16. Zhang W, Guo Y, Yu S, Wei J, Jin J. Effects of edaravone on the expression of $\beta$-defensin- 2 mRNA in lung tissue of rats with myocardial ischemia reperfusion. Mol Med Rep. 2013;7(5):1683-1687.

17. Tsujimoto I, Hikoso S, Yamaguchi O, et al. The antioxidant edaravone attenuates pressure overload-induced left ventricular hypertrophy. Hypertension. 2005;45(5):921-926.

18. Arumugam S, Thandavarayan RA, Veeraveedu PT, et al. Beneficial effects of edaravone, a novel antioxidant, in rats with dilated cardiomyopathy. J Cell Mol Med. 2012;16(9):2176-2185.

19. Liao Y, Ishikura F, Beppu S, et al. Echocardiographic assessment of LV hypertrophy and function in aortic-banded mice: necropsy validation. Am J Physiol. 2002;282(5):H1703-H1708.

20. Zhao ZQ, Puskas JD, Xu D, et al. Improvement in cardiac function with small intestine extracellular matrix is associated with recruitment of c-kit cells, myofibroblasts, and macrophages after myocardial infarction. J Am Coll Cardiol. 2010;55(12):1250-1261.

21. Garg S, Drazner MH. Refining the classification of left ventricular hypertrophy to provide new insights into the progression from hypertension to heart failure. Curr Opin Cardiol. 2016;31(4):387-393.

22. Balakumar P, Jagadeesh G. Structural determinants for binding, activation, and functional selectivity of the angiotensin AT1 receptor. $J \mathrm{Mol}$ Endocrinol. 2014;53(2):R71-R92.
23. Guo L, Yin A, Zhang Q, Zhong T, O'Rourke ST, Sun C. Angiotensin(1-7) attenuates angiotensin II-induced cardiac hypertrophy via a Sirt3dependent mechanism. Am J Physiol. 2017;312(5):H980-H991.

24. Nguyen Dinh Cat A, Montezano AC, Burger D, Touyz RM. Angiotensin II, NADPH oxidase, and redox signaling in the vasculature. Antioxid Redox Signal. 2013;19(10):1110-1120.

25. Imayama I, Ichiki $\mathrm{T}$, Inanaga $\mathrm{K}$, et al. Telmisartan downregulates angiotensin II type 1 receptor through activation of peroxisome proliferatoractivated receptor gamma. Cardiovasc Res. 2006;72(1):184-190.

26. Wermuth PJ, Jimenez SA. The significance of macrophage polarization subtypes for animal models of tissue fibrosis and human fibrotic diseases. Clin Transl Med. 2015;4:2.

27. Koka V, Xiao RH, Chung ACK, Wang W, Luan DT, Hui YL. Angiotensin II up-regulates angiotensin I-converting enzyme (ACE), but down-regulates ACE2 via the AT1-ERK/p38 MAP kinase pathway. Am J Pathol. 2008;172(5):1174-1183.

28. Patel VB, Zhong JC, Grant MB, Oudit GY. Role of the ACE2/ angiotensin 1-7 axis of the renin-angiotensin system in heart failure. Circ Res. 2016;118(8):1313-1326.

29. Wang NP, Erskine J, Zhang WW, et al. Recruitment of macrophages from the spleen contributes to myocardial fibrosis and hypertension induced by angiotensin II. J Renin Angiotensin Aldosterone Syst. 2017; 18(2):1-14.

30. Kain D, Amit U, Yagil C, et al. Macrophages dictate the progression and manifestation of hypertensive heart disease. Int J Cardiol. 2016;203: 381-395.

31. Hunyady L, Catt KJ. Pleiotropic AT1 receptor signaling pathways mediating physiological and pathogenic actions of angiotensin II. Mol Endocrinol. 2006;20(5):953-970.

32. Zhang W, Chen DQ, Qi F, Wang J, Xiao WY, Zhu WZ. Inhibition of calcium-calmodulin-dependent kinase II suppresses cardiac fibroblast proliferation and extracellular matrix secretion. J Cardiovasc Pharmacol. 2010;55(1):96-105.

33. Lei B, Hitomi H, Mori T, et al. Effect of efonidipine on TGF- $\beta 1$-induced cardiac fibrosis through Smad2-dependent pathway in rat cardiac fibroblasts. J Pharmacol Sci. 2011;117(2):98-105.

34. Yuan SM, Jing H. Cardiac pathologies in relation to Smad-dependent pathways. Interact Cardiovasc Thorac Surg. 2010;11(4):455-460.

35. Wang B, Omar A, Angelovska T, et al. Regulation of collagen synthesis by inhibitory Smad7 in cardiac myofibroblasts. Am J Physiol. 2007; 293(2):H1282-H1290.

36. Katz AM, Rolett EL. Heart failure: when form fails to follow function. Eur Heart J. 2016;37(5):449-454.

37. Szymanski MK, Buikema JH, van Veldhuisen DJ, et al. Increased cardiovascular risk in rats with primary renal dysfunction; mediating role for vascular endothelial function. Basic Res Cardiol. 2012; 107(2):242-256.

38. Savinova OV, Gerdes AM. Myocyte changes in heart failure. Heart Fail Clin. 2012;8(1):1-6.

39. Matsumoto T, Wada A, Tsutamoto T, Ohnishi M, Isono T, Kinoshita M. Chymase inhibition prevents cardiac fibrosis and improves diastolic dysfunction in the progression of heart failure. Circulation. 2003; 107(20):2555-2558.

40. Ryoke T, Gu Y, Mao L, et al. Progressive cardiac dysfunction and fibrosis in cardiomyopathic hamster and effects of growth hormone and angiotensin-converting enzyme inhibition. Circulation. 1999; 100(16):1734-1743.

41. Lymperopoulos A, Sturchler E, Bathgate-Siryk A, et al. Different potencies of angiotensin receptor blockers at suppressing adrenal $\beta$-Arrestin1-dependent post-myocardial infarction hyperaldosteronism. J Am Coll Cardiol. 2014;64(25):2805-2806.

42. AbdAlla S, Lother H, Abdel-tawab AM, Quitterer U. The angiotensin II AT2 receptor is an AT1 receptor antagonist. J Biol Chem. 2001; 276(43):39721-39726.

43. Dabul S, Bathgate-Siryk A, Valero TR, et al. Suppression of adrenal $\beta$-arrestin1-dependent aldosterone production by ARBs: head-to-head comparison. Sci Rep. 2015;5:8116. 
44. Chou CH, Chen YH, Hung CS, et al. Aldosterone impairs vascular smooth muscle function: from clinical to bench research. J Clin Endocrinol Metab. 2015;100(11):4339-4347.

45. Nappi JM, Sieg A. Aldosterone and aldosterone receptor antagonists in patients with chronic heart failure. Vasc Health Risk Manag. 2011; $7: 353-363$
46. Medscape. FDA approves edaravone (Radicava) for amyotrophic lateral sclerosis. Available from: http://www.medscape.com/ viewarticle/879641. Accessed May 5, 2017.

47. National Research Council. Guide for the Care and Use of Laboratory Animals: Eighth Edition. Washington DC, USA: The National Academies Press; 2011.

\section{Publish your work in this journal}

Drug Design, Development and Therapy is an international, peerreviewed open-access journal that spans the spectrum of drug design and development through to clinical applications. Clinical outcomes, patient safety, and programs for the development and effective, safe, and sustained use of medicines are the features of the journal, which has also been accepted for indexing on PubMed Central. The manuscript management system is completely online and includes a very quick and fair peer-review system, which is all easy to use. Visit http://www.dovepress.com/testimonials.php to read real quotes from published authors.

Submit your manuscript here: http://www.dovepress.com/drug-design-development-and-therapy-journal 